\title{
GvHD Lung Symptom Score
}

National Cancer Institute

\section{Source}

National Cancer Institute. GvHD Lung Symptom Score. NCI Thesaurus. Code C131049.

A score for graft versus host disease based on the severity of lung symptoms. 